ISSN 2223-7747

www.mdpi.com/journal/plants

Article

\title{
Phosphorylation Affects DNA-Binding of the Senescence- Regulating bZIP Transcription Factor GBF1
}

\author{
Anja Smykowski, Stefan M. Fischer and Ulrike Zentgraf *
}

ZMBP, General Genetics, University of Tuebingen, Auf der Morgenstelle 32, Tuebingen 72076, Germany; E-Mails: smyka@gmx.net (A.S.); stefan.fischer@zmbp.uni-tuebingen.de (S.M.F.)

* Author to whom correspondence should be addressed; E-Mail: ulrike.zentgraf@zmbp.unituebingen.de.

Academic Editor: Salma Balazadeh

Received: 8 July 2015 / Accepted: 8 September 2015 / Published: 16 September 2015

\begin{abstract}
Massive changes in the transcriptome of Arabidopsis thaliana during onset and progression of leaf senescence imply a central role for transcription factors. While many transcription factors are themselves up- or down-regulated during senescence, the bZIP transcription factor G-box-binding factor 1 (GBF1/bZIP41) is constitutively expressed in Arabidopsis leaf tissue but at the same time triggers the onset of leaf senescence, suggesting posttranscriptional mechanisms for senescence-specific GBF1 activation. Here we show that GBF1 is phosphorylated by the threonine/serine CASEIN KINASE II (CKII) in vitro and that CKII phosphorylation had a negative effect on GBF1 DNA-binding to G-boxes of two direct target genes, CATALASE2 and RBSCS1a. Phosphorylation mimicry at three serine positions in the basic region of GBF1 also had a negative effect on DNA-binding. Kinase assays revealed that CKII phosphorylates at least one serine in the basic domain but has additional phosphorylation sites outside this domain. Two different ckII $\alpha$ subunit 1 and one $\alpha$ subunit 2 T-DNA insertion lines showed no visible senescence phenotype, but in all lines the expression of the senescence marker gene $S A G 12$ was remarkably diminished. A model is presented suggesting that senescence-specific GBF1 activation might be achieved by lowering the phosphorylation of GBF1 by CKII.
\end{abstract}

Keywords: senescence regulation; GBF1; CASEIN KINASE II; phosphorylation; phosphorylation mimicry 


\section{Introduction}

Onset and progression of leaf senescence is accompanied by a massive change in the transcriptome, implying an important role of transcription factors in this process [1-4]. A temporal transcript profiling using microarrays with high resolution covering 22 time points of a defined single leaf of Arabidopsis during onset and progression of leaf senescence revealed a distinct chronology of processes. Promoter motif and transcription factor analyses revealed a differential activation of regulatory genes that influence gene expression at different time points during senescence progression [4]. Obviously, the two transcription factor families, WRKY and NAC factors, which largely expanded in the plant kingdom are both overrepresented in the senescence transcriptome of Arabidopsis and several members of both families have already been characterized as playing important roles in senescence regulation in Arabidopsis and other plant species [5-14]. Family members of both groups have been shown to react to elevated levels of reactive oxygen species (ROS), especially hydrogen peroxide at the transcriptional level, and are at the same time involved in regulating the intracellular concentrations of these molecules [7-11,14]. Remarkably, response to ROS was one of the earliest features identified in the high-resolution temporal chronology of processes [4].

Consistent with this observation, a tight regulation of the activity of the hydrogen peroxide scavenging enzymes catalase (CAT) and ascorbate peroxidase (APX) were detected in Arabidopsis, rapeseed and sunflower plants leading to elevated levels of hydrogen peroxide during bolting and flowering time, which is achieved on either the transcriptional or posttranscriptional level [15-18]. In Arabidopsis plants, transcriptional repression of $C A T 2$ by the bZIP transcription factor GBF1 at bolting time appears to be the initial step for elevation of hydrogen peroxide contents, whereas the loss of APX1 activity is not achieved on the transcriptional level. Inhibition of the APX activity is most likely mediated through its own substrate $\mathrm{H}_{2} \mathrm{O}_{2}$ in which APX is sensitive to its own substrate exactly in this developmental time window. APX regulation is therefore believed to build a positive feed-back loop to enhance the increase of hydrogen peroxide at this time point of development $[15,18]$. If transcriptional repression of $C A T 2$ is abolished in $g b f 1$ mutant plants, no elevation of the hydrogen peroxide concentration is seen, and consequently a delay in leaf senescence was observed [19]. However, expression analyses revealed that GBF1 appears to be constitutively expressed in leaf tissue [19] suggesting posttranscriptional mechanisms for senescence-specific GBF1 regulation. GBF1 belongs to the bZIP family of transcription factors, which consists of at least 75 bZIP members in Arabidopsis divided into 10 subgroups based on sequence similarity of the basic region and the presence of additional conserved motifs. These bZIP transcription factors play crucial roles in almost every response pathway, including light and stress signaling, pathogen defense, seed maturation and flower development [20]. Plant bZIP factors preferentially interact with cis-elements containing an ACGT core sequence, which can be found in C-and G-boxes [21,22]. The G-boxes drive gene expression in response to various exogenous triggers like light, anaerobiosis and endogenous signals such as abscisic acid or methyl-jasmonate [23].

bZIP factor activity is altered by different mechanisms, one of which is phosphorylation. Phosphorylation can stabilize proteins and diminish degradation, as in the case of HY5, a G-box binding factor of the H group [24]. Most bZIPs hold putative phosphorylation sites not only in their DNA-binding domain but also in the nuclear localization site, or in the case of GBF1, also in the multifunctional mosaic region $[25,26]$. Furthermore, it is known that a few bZIP factors, for example GBF2, are translocated 
into the nucleus upon light treatment. This translocation might be due to phosphorylation triggered by various signals [27]. Concerning the DNA-binding of transcription factors, phosphorylation has been shown to stimulate [28,29], abolish [30,31] or simply not affect the interaction of DNA and protein [32].

GBF1 in particular has been shown to be phosphorylated by a partially purified serine/threonine kinase of broccoli with many characteristics of CASEIN KINASE II (CKII) and the phosphorylation by this CKII-like protein was exclusively mapped to serines and had an enhancing effect on DNA-binding in vitro [28]. However, phosphorylation mimicry often had a negative effect on DNA binding of bZIP factors [25,31]. Meshi and coworkers were also able to show that the basic region of HPB-1a of wheat, which contains the same amino acid sequence in the basic region as GBF1, is phosphorylated mainly through a calcium-dependent protein kinase and this negatively modulated DNA-binding. Other regions outside the basic region of HPB-1a were also phosphorylated by CKII [31].

CKII $\alpha$ subunits affect multiple developmental and stress-responsive pathways in Arabidopsis including light signaling, flowering time and the circadian clock [33-35]. In plants, both $\alpha$ and $\beta$ subunits of CKII are often encoded by multiple genes. In Arabidopsis thaliana, each subunit is encoded by four genes. While three of these slightly different $\alpha$ subunits localize to the cytoplasm and/or the nucleus where they mainly phosphorylate transcription factors, one $\alpha$ subunit $(\alpha 4)$ is targeted to the chloroplast [36] and phosphorylates a plastid sigma factor (AtSIG6) [37]. At least 37 direct targets of CKII have already been identified in plants (for overview see [35]) but besides the bZIP factor GBF1, no other target indicates the involvement of CKII in senescence regulation. However, CKII has been shown to be involved in SA mediated phosphorylation of TGA2, another bZIP transcription factor, which also resulted in significantly reduced DNA binding in vitro [38]. Moreover, CKII is also involved in ABA responses in maize and Arabidopsis [39], and both hormones have been shown to be involved in early senescence regulation $[3,8,10]$. To test whether CKII is involved in senescence-specific activation of GBF1, we performed kinase assays with wild type and mutated GBF1. Since CKII is highly conserved throughout eukaryotes, we used mammalian CKII which could phosphorylate GBF1 and its substrate calf casein in vitro. Mutation of all serines in the DNA-binding basic domain (position $-11 /-15 /-19$ ) led to a much weaker phosphorylation signal compared to wild type GBF1 indicating that at least one of these serines is targeted by CKII but also means that other CKII phosphorylation sites exist outside the basic domain. Plant CKII $\alpha$ subunit addition as well as phosphorylation mimicry led to a reduced DNAbinding to G-boxes in in vitro binding assays to promoter fragments of two direct target genes, CAT2 and RBCS1a. ckII $\alpha$ subunit1 and -2 single mutants were analyzed for their senescence phenotype but no visible phenotype could be observed; however, expression of the senescence marker gene SAG12 was clearly delayed in all mutant lines.

\section{Results}

\subsection{GBF1 Is Phosphorylated by CKII}

Klimczak and coworkers were able to show phosphorylation of GBF1 by a CASEIN KINASE II-like protein complex isolated directly from broccoli [28]. CKIIs are highly conserved among eukaryotes and subunits can complement each other [40]. Maize subunits, for instance, have been shown to assemble with human CKII subunits to functional oligomeric forms [41]. Therefore, an in vitro kinase assay was 
conducted using a commercially available mammalian CKII (NEB). The mammalian CKII was able to phosphorylate recombinant GBF1 as well as its native substrate casein (Figure 1A). Phosphorylation of GBF1 was not detected without the addition of CKII or $\left[\gamma_{-}{ }^{32} \mathrm{P}\right]-\mathrm{ATP}$. An additional band not corresponding to GBF1 is visible in all lanes, even where CKII is missing. This appears to be a protein phosphorylated by a bacterial kinase and can be designated as background signal of the raw BL21 bacterial lysate in this assay. The in vitro kinase assay was repeated using purified $6 \times$ His-tagged GBF 1 protein (Figure 1B) and phosphorylation of mutated versions of GBF1 where all serines (position $-11 /-15 /-19)$ in the basic domain were changed to aspartate. These three serines are highly conserved in nearly all plant bZIPs, and structural modelling revealed that these serines are in direct physical contact with the DNA [25]. The triple mutant version of GBF1 still showed a weak signal, demonstrating that CKII phosphorylates the basic domain as the signal is reduced but additional phosphorylation sites have to be present outside the basic domain as radioactivity is still incorporated.
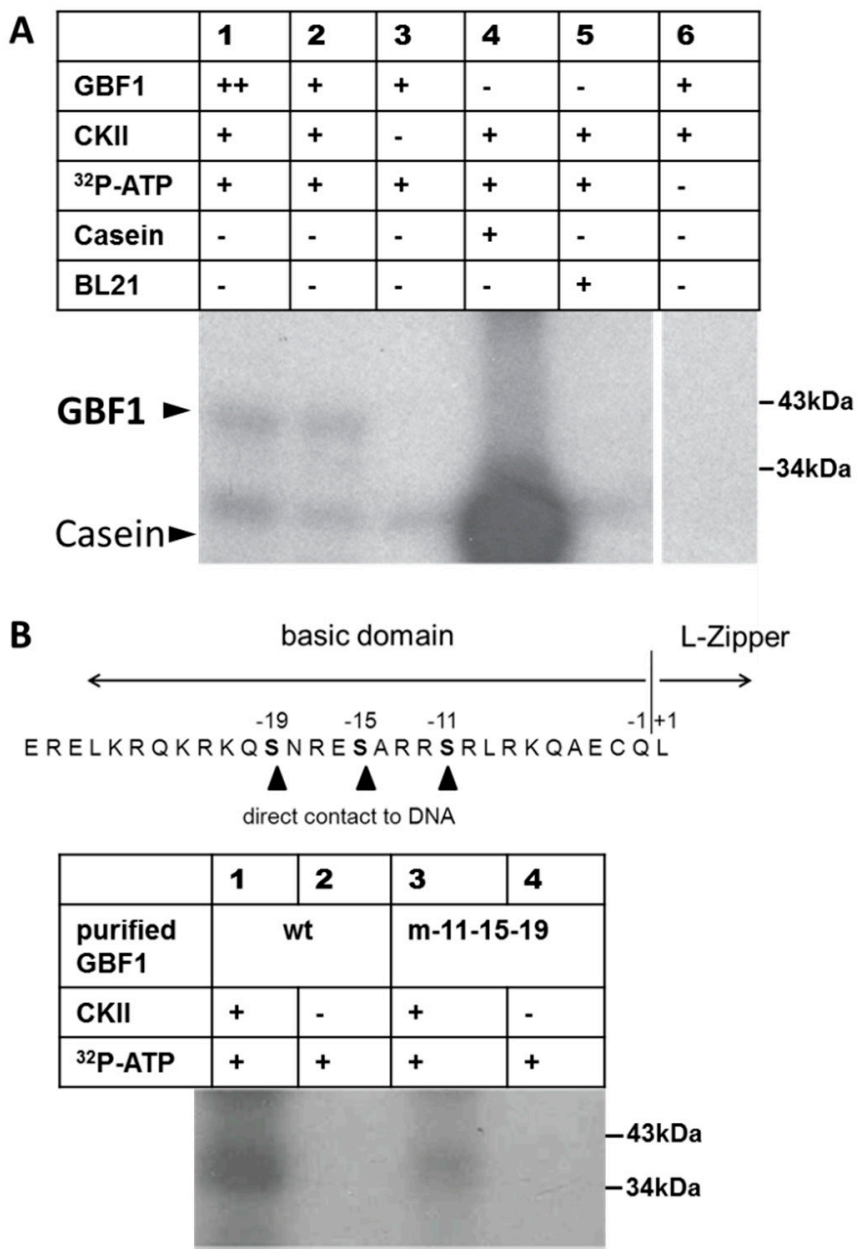

Figure 1. In vitro kinase assay with mammalian CKII. (A) E. coli BL21 raw lysate expressing recombinant GBF1 or no recombinant protein (BL21, lane 5) incubated with or without mammalian Casein Kinase II (NEB). Calf casein (Qiagen) was used as control substrate. Size in $\mathrm{kDa}$ is indicated on the right. $++: 45 \mu \mathrm{g},+: 15 \mu \mathrm{g}$ raw protein lysate. All probes contained unlabeled ATP $(100 \mu \mathrm{mol})$; (B) Purified $6 \times$ His-tagged GBF1 protein (wt) or mutated at position $-11 /-15 /-19$ of the basic domain $(m-11-15-19)$ incubated with or without mammalian CKII (NEB). 


\subsection{GBF1 DNA-Binding Is Altered through Phosphorylation by CKII or Phosphorylation Mimicry}

DNA-binding assays were performed in the presence of CKII or with mutated versions of GBF1 in order to characterize the importance of phosphorylation of the GBF1 basic domain for its DNA-binding activity. An ELISA-based DNA-binding assay was established to analyze the DNA-binding of GBF1 to CAT2 and RBCS1a promoter fragments, two direct target genes of GBF1 [19,42]. Biotinylated oligonucleotides containing either the G-box or mutated versions of the G-box of the CAT2 promoter or of the RBCS1 a promoter were attached to streptavidin coated ELISA wells, respectively. These plates were incubated with crude protein extracts of bacteria expressing recombinant $6 \times$ His-tagged GBF1 in different dilutions. DNA-bound GBF1 was detected using Anti-His-antibodies conjugated with horse radish peroxidase and the substrate 1,2-phenylenediamine hydrochloride. G-Boxes of both promoters were bound by recombinant GBF1 in a concentration dependent manner whereas binding to the mutated versions of the G-Box was very restricted (Figure 2A). If CKII was added prior to the binding assay either as purified mammalian CKII or as recombinant plant CKII 2 2, DNA-binding was diminished, indicating that phosphorylation by CKII has a negative effect on DNA-binding while addition of plant CKII alone without GBF1 had no effect (Figure 2B,C).

To investigate the role of the different phosphorylation sites in the DNA-binding domain, the three serines of this domain were mutated stepwise by site-directed mutagenesis to aspartate to mimic phosphorylation or to alanine to mimic the non-phosphorylated state. Successful mutagenesis was verified by sequencing the mutated plasmids. Single, double, and triple mutated versions were tested for their ability to bind to DNA. Raw extracts of E. coli BL21 cells expressing the different recombinant GBF1 versions were used in DPI-ELISAs with G-boxes of the CAT2 and RBSCla promoters, respectively. Expression of the $6 \times$ His-tagged recombinant proteins were analyzed by western blots (Figure S1), the amounts of recombinant protein in the raw lysates were quantified using ImageQuantTL software, and DNA-binding was normalized to the protein amounts (Figure 3). All exchanges to aspartate led to a significant decrease of the DNA-binding ability of GBF1 except of the S-19D exchange, which led to non-significant decrease of binding to both promoter fragments. If the same amino acids were changed to alanine significant changes were only observed in DNA-binding for the S-19A mutant version and the CAT2 fragment. The S-11A/S-15A double mutant showed increased DNA-binding (not significant) but in this case the protein amount of the recombinant protein in the raw lysates was extremely high. Thus the increase in DNA-binding is most likely due to underestimated amounts of protein by the software leading to overestimated DNA-binding values. Taken together, the exchange of serine to aspartate has a clear inhibitory effect on DNA-binding, whereas the exchange to alanine has only moderate, non-significant effects. Since the exchange to aspartate introduces a negative charge to mimic phosphorylation, actual phosphorylation of the serines in the DNA-binding domain most likely leads to the inhibition of DNA-binding in which serine at position -11 is most and serine at position -19 least effective. The S-x-x-E/D-pS consensus binding motif of CKII was present only at position -15 but according to the analyses of CKII substrates of Meggio and Pinna [43] all three serines are in the range of possible targets for CKII phosphorylation (Figure S2). For the serine at position -19 also the exchange to alanine led to a significant decrease in DNA-binding, at least with the CAT2 promoter fragment, possibly indicating that a mutation at this position in general leads to an inhibitory effect on DNA binding. 
A
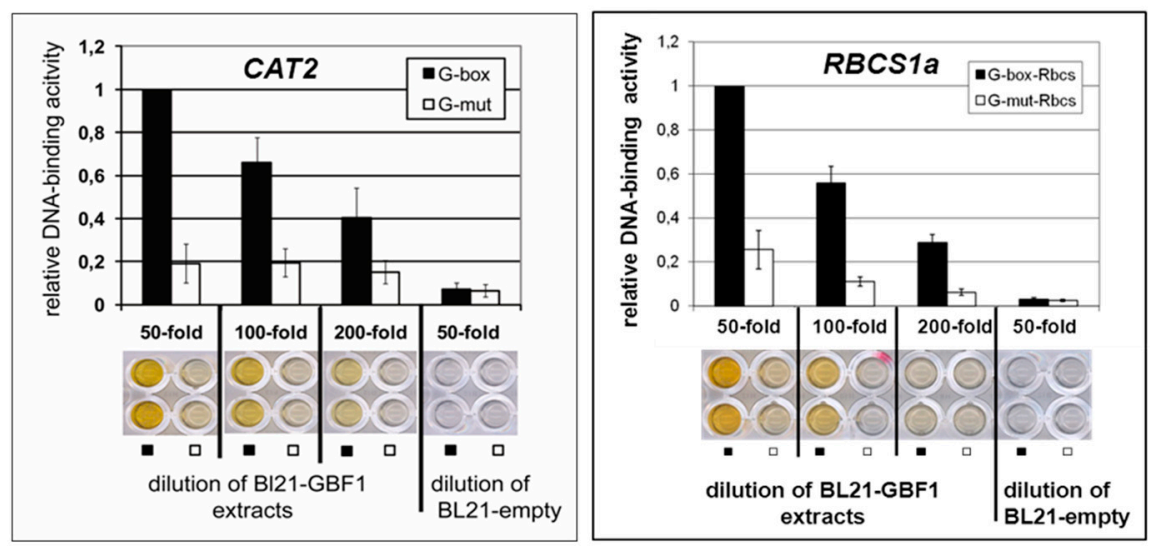

B
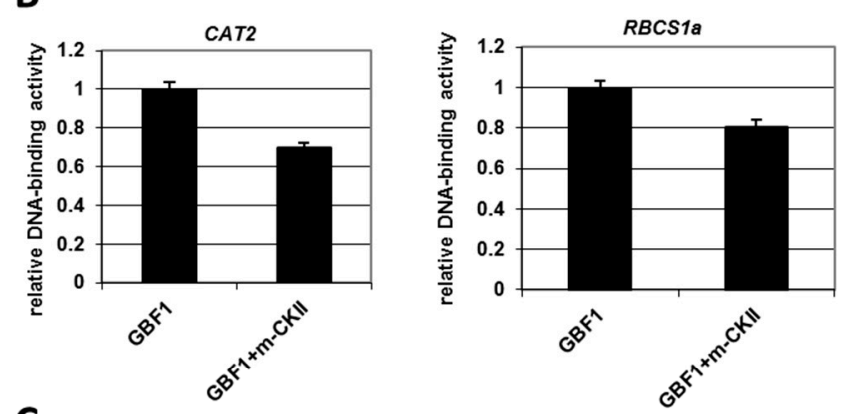

C
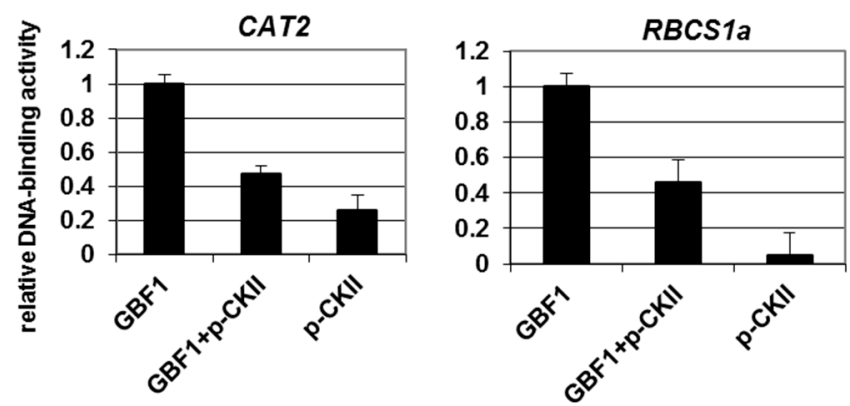

Figure 2. DPI-ELISA using recombinant GBF1 with the G-box of the CAT2 and the RBCS1 $a$ promoter. Biotinylated plate coupled DNA fragments containing either the G-box (black bars) or a mutated version (G-mut; white bars) were incubated with crude E. coli BL21 extracts expressing recombinant $6 \times$ His-tagged GBF1 in different dilutions or the empty vector. Colorimetric reaction was quantified with a plate reader and plates were also photographed. The interactions between 50-fold diluted crude extracts and the G-box were set to 1.0, and all other interactions are expressed as relative values referring to GBF1/G-Box. (A) Concentration-dependent DNA-binding reaction of crude $E$. coli BL21 extracts expressing recombinant $6 \times$ His-tagged GBF 1 with the G-box containing fragment of the $C A T 2$ promoter (left) or of the RBCSIa promoter (right); (B) DPI-ELISA after pre-incubation with or without mammalian CKII (m-CKII); (C) DPI-ELISA after pre-incubation with or without plant CKII $\alpha$ subunit 2 expressed in E. coli (p-CKII). Error bars indicate SE (A) or SD (B and C) of 2-6 independent experiments. 


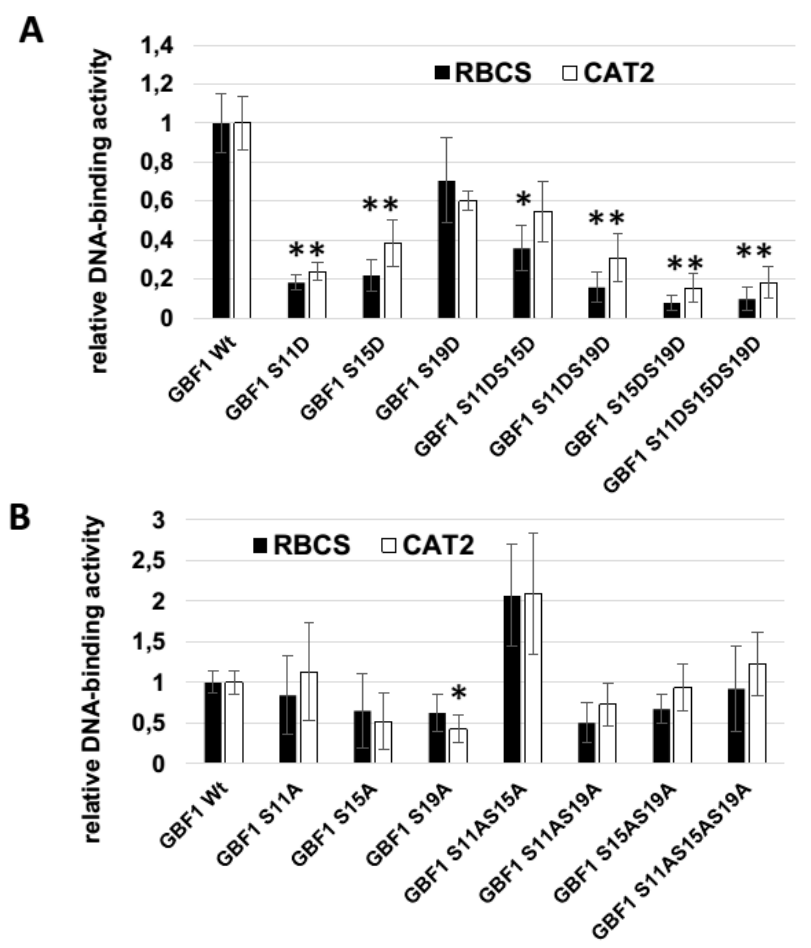

Figure 3. DPI-ELISA using recombinant GBF1 and single, double, and triple mutants of the serines in the basic domain with the G-boxes of the CAT2 and the RBCSIa promoter. Biotinylated plate coupled DNA fragments containing the G-box of the RBCSIa promoter (black bars) or of the CAT2 promoter (white bars) were incubated with crude E. coli BL21 extracts expressing recombinant $6 \times$ His-tagged GBF1 or GBF1 with substituted serines of the basic domain. The amounts of recombinant protein in the raw lysates were quantified on a Western blot using ImageQuantTL software, and DNA-binding was normalized to the protein amounts. The interactions between 50-fold diluted crude extracts and the wild type GBF1 was set to 1.0, all other interaction are expressed as relative values referring to GBF1/G-Box. (A) Consecutive substitution of serines at position $-11,-15$, and -19 with aspartate (D); (B) consecutive substitution of serines (S) at position $-11,-15$, and -19 with alanine (A). Error bars indicate standard deviation of 3-4 independent experiments, significant differences compared to wild type GBF1 are marked with asterisks $(t$-test, $p<0.001)$.

\subsection{Senescence Phenotype of ckII Mutant Lines}

In order to analyze whether GBF1 phosphorylation via CKII has an effect on the progression of leaf senescence in planta, we characterized two different ckIIal and one ckIIa2 T-DNA insertion lines. T-DNA insertions were localized in exon or intron sequences in the CKII 1 gene, respectively, and in an exon in the CKIIa2 gene. Plants which were homozygous for these insertions were identified by a

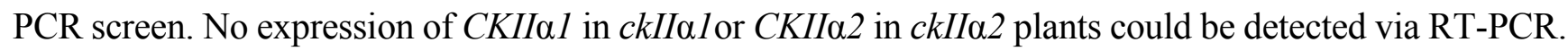

These lines were grown side by side with wild type plants under long-day conditions for senescence phenotyping. Leaves of at least five plants were categorized into 4 groups according to their leaf color (fully green, green/yellow, fully yellow and brown/dry) (Figure 4A). A picture of a typical example of leaves of 7-week-old plants sorted with the help of a color code according to their age is presented in Figure S3. Furthermore, the chlorophyll contents of leaves No. 4 to 7 were measured by using an 
atLEAF+ chlorophyll meter (Figure 4B). In comparison to wild type plants, ckII $\alpha$ mutants showed no clear visible phenotype according to the sorted leaves and the percentage of leaves in the different categories. However, a delay in chlorophyll loss could be observed in ckIIa2 mutants, and expression of $S A G 12$ was clearly reduced in all mutant plants of the same age compared to WT (Figure 4C) suggesting a delay in senescence on the molecular level. CAT2 and RBCS1a expression was also tested in 4- and 6-week-old plants, being the time point when these two target genes of GBF1 are downregulated in wild type plants and the transcription factor WRKY53 is upregulated. Whereas RBCS1a and WRKY53 expression was not significantly changed in both mutant lines compared to wild type plants, CAT2 downregulation was impaired in the ckII 2 mutant line, indicating that the different $\alpha$ subunit genes most likely have overlapping but also non-redundant functions (Figure S6). Moreover, intracellular hydrogen peroxide contents were measured in these plants lines over development in which the increase in $\mathrm{H}_{2} \mathrm{O}_{2}$ was slowed down in the ckIIa2 mutant line (Figure S7).

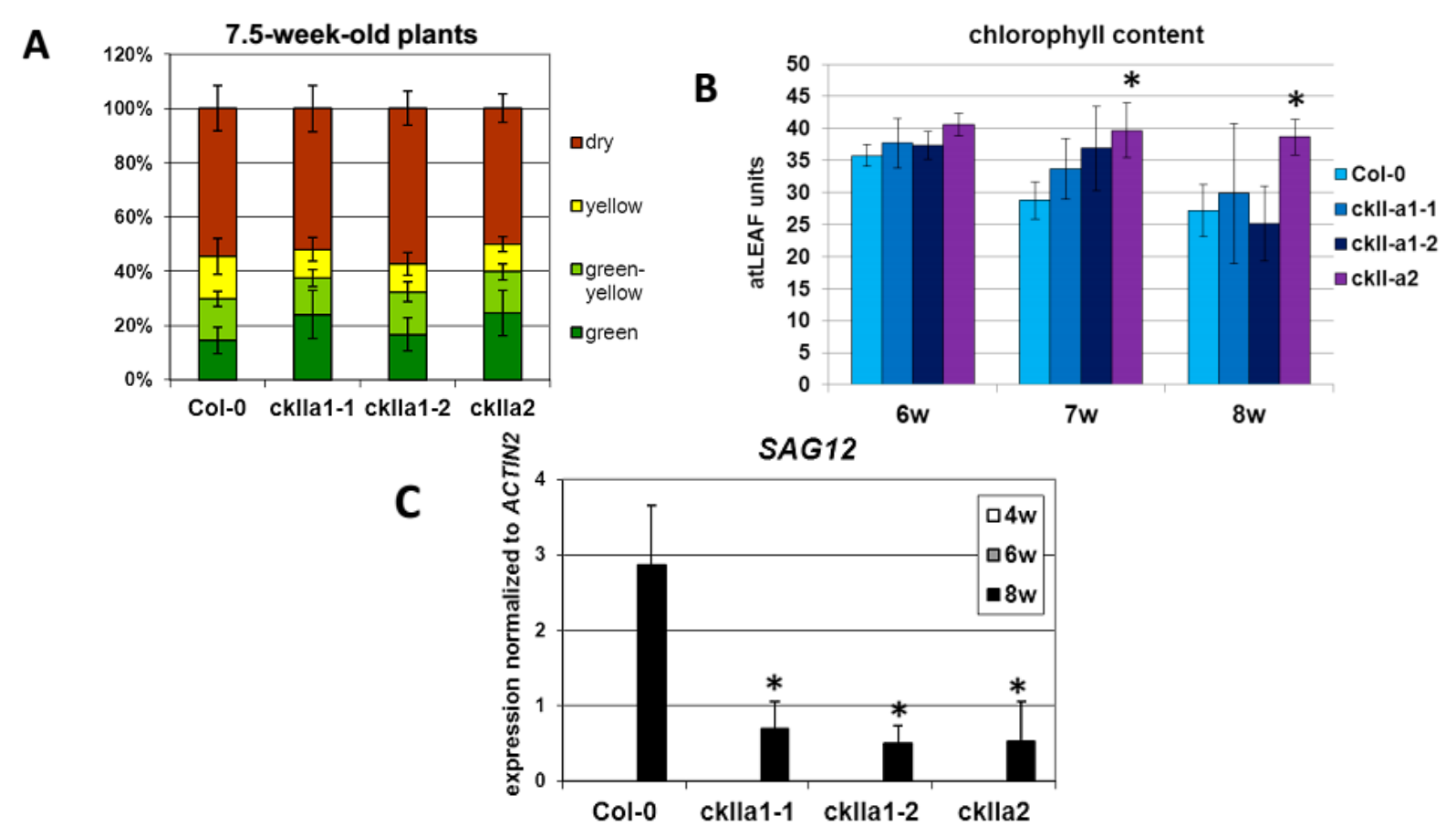

Figure 4. Senescence phenotypes of wild type Col-0, ckII 1 and ckII 2 mutant plants. Four plant lines were analyzed over development. (A) For a quantitative evaluation of leaf senescence, leaves of at least five plants were categorized in four categories according to their leaf color: (1) "green"; (2) leaves starting to get yellow from the tip as "yellow-green"; (3) completely yellow leaves as "yellow" and (4) dry and/or brown leaves as "dry". The percentages of each group with respect to total leaf numbers are presented. (B) Chlorophyll content was measured using an atLEAF+ chlorophyll meter over development in leaves No. 4 to 7 of 6-, 7- and 8-week-old plants. Error bars indicate standard deviations of at least five plants. (C) Expression of the senescence marker gene SAG12 encoding a cysteine protease was analyzed in leaf No. 6 and 7 of 4-. 6- and 8-week-old plant material by RT-PCR and normalized to the expression of ACTIN2. Error bars indicate standard deviations of four independent experiments, significant differences compared to wild type plants are marked with asterisks $(t$-test, $p<0.05)$. 


\section{Discussion}

Regulation of gene expression is the major regulatory mechanism underlying onset and progression of senescence implying a central role for transcription factors. Many of these are in turn regulated on the transcriptional level, but at some point specific factors have to be activated in a transcription-independent manner [1-5]. GBF1 appears to be one of these factors, since expression of GBF1 is not altered in leaf tissue over development. GBF1 was shown to regulate the onset of leaf senescence most likely through the transcriptional down-regulation of the genes encoding the hydrogen peroxide scavenging enzyme $C A T 2$ and the small subunit of RUBISCO (RBCS1a). gbf1 mutant plants maintained constant CAT2 expression, thus lacking an increase in intracellular hydrogen peroxide and the induction of $S A G S$ and consequently exhibited a delayed senescence phenotype [19].

One possible mode of posttranscriptional regulation is phosphorylation of transcription factors at specific sites leading to activation or inhibition of their activity. Klimczak and coworkers [28] partially purified a GBF1 phosphorylating activity of broccoli by chromatography on heparin-sepharose and DEAE-cellulose and characterized essential features of recombinant Arabidopsis CKII activity [28]. Reconstitution of Arabidopsis CKII from recombinant subunits to a tetrameric complex had properties very similar to those of the oligomeric CKII-like complex of broccoli, whereas the properties of the catalytic CKII $\alpha 1$ subunit alone differed [44]. Since coexpression of the $\alpha$ and $\beta$ subunits in E. coli lead to massive formation of inclusion bodies [44], we were not able to solubilize and reconstitute active tetrameric complexes. Since CKIIs are highly conserved among eukaryotes and subunits can complement each other [40], we verified GBF1 in vitro phosphorylation using mammalian CKII (Figure 1). Moreover, mutation of all serines in the DNA-binding basic domain (position $-11 /-15 /-19$, Figure 1B) led to a much weaker phosphorylation signal compared to wild type GBF1, indicating that CKII phosphorylation takes place in the DNA-binding domain but additional CKII phosphorylation sites appear to exist outside the basic domain, possibly in the proline-rich domain controlling gene transcriptional regulation or in the multifunctional mosaic region $[25,26]$.

In order to see whether phosphorylation has an effect on GBF1 DNA-binding activity, we performed DNA-binding analyses in the presence of mammalian CKII and recombinant plant CKII 22 subunits to both CAT2 and RBCSIa promoter fragments (Figure 2). In animal cells, phosphorylation by CKII can inhibit the DNA-binding activity of transcription factor homodimers but not heterodimers [45]. Here, the addition of mammalian CKII as well as of a recombinant plant CKIIa2 subunit to GBF1 DNA-binding assays also inhibited DNA-binding. Phosphorylation mimicry by exchange of the serines to aspartate in the DNA-binding domain also revealed that DNA-binding of pseudo-phosphorylated GBF1 is always diminished but the degree depends on the site of phosphorylation and on the G-box it is binding to (Figure 3). The mutation of serine at position -11 seemed to be most effective as single mutant while $-15-19$ double mutants and the triple mutant had the severest effect on DNA-binding to both CAT2 and $R B C S 1$ a promoter fragments. Yet, serine at position -15 is the only serine which is surrounded by a motif identical to the CKII consensus binding sequence (Figure S2), but all three serines are possible CKII sites according to the CKII binding motif analyses of Meggio and Pinna [43]. Thus, GBF1 activity is most likely regulated through the interplay of different kinases which might also have different effects on DNA-binding. Exchange of the serines to alanine had no significant effect on the DNA binding except for serine at position -19 and the CAT2 promoter fragment (Figure 3) suggesting that (i) the effect of 
exchanges to aspartate is most likely due to a phosphorylation mimicry and not a general effect of the mutation and (ii) the serine in position -19 appears to be more important for the overall structure of the GBF1 protein.

While Meshi and coworkers [31] claimed that phosphorylation of the basic region of the bZIP factor HBP1a of wheat which contains the same amino acid sequence in the basic region as GBF1 through calcium dependent kinases leads to a decrease in DNA-binding and Berberich and Cole could show that Casein kinase II inhibits the DNA-binding activity of Max homodimers [45], Klimczak and coworkers [28] clearly demonstrated that phosphorylation through CKII-like protein complexes isolated from broccoli increased DNA-binding of the Arabidopsis GBF1. Therefore, it is still unclear which effect phosphorylation of the basic domain has on bZIP function. Our results suggest that CKII phosphorylation using purified commercially available mammalian CKII protein as well as recombinant Arabidopsis CKII $\alpha 2$ subunits has a negative effect on DNA-binding of GBF1 to the promoter fragments of two direct target genes of GBF1, CAT2 and RBCS1a (Figure 2). Phosphorylation mimicry by exchange of the three serines at positions $-11,-15$, and -19 of the DNA-binding domain for which structural analyses proposed a direct contact to DNA [25] also led to a decrease in DNA-binding activity (Figure 3). This might be due to an inhibition of dimer formation of GBF1, which is a general prerequisite for bZIP activity. Even though phosphorylation mimicry was introduced only in the DNA-binding domain, DNA-binding and dimerization are two coupled processes influencing each other. Whether lower DNA-binding of phosphorylated monomers to DNA inhibits effective dimer formation or inhibited dimer formation between phosphorylated proteins inhibits DNA-binding still has to be elucidated. There is evidence for both models, dimerization can be observed either as protein-protein interaction without DNA [46] or dimer formation is initiated after sequential binding of the monomers to the DNA [47,48]. The monomer pathway allows faster assembly of certain bZIP dimer-DNA complexes and provides an efficient means of discriminating between specific and nonspecific DNA target sites [47].

CKII plays a critical role in various physiological processes such as light signaling, circadian rhythms, hormone responses, and flowering time. CKII is not regulated on the transcriptional level (Figure S5) but appears to be a housekeeping kinase which is also involved in very basic cellular processes like the cell cycle, DNA damage or translation, and is therefore also not regulated by a simple on/off switch but more in a dynamic way in which the activity of the catalytic CKII $\alpha$ subunit is modulated by the regulatory $\beta$ subunits [35].

In order to characterize whether CKII could be a candidate for the senescence-specific activation of GBF1, ckII $\alpha$ mutants were analyzed (Figure 4). In contrast to the well-defined tetrameric structure of animal and yeast CKIIs, plant CKII can also exists in a monomeric and an oligomeric form whose subunit composition is not well defined. The $\alpha$-subunits are the catalytic subunits and are sufficient for the phosphorylation of GBF1 [44]. Therefore, T-DNA insertion lines for the two $\alpha$-subunits, CKII 1 (At5g67380) and CKIIa2 (At3g50000), which are mainly expressed in leaves [36] were used to characterize the role of CKII in senescence regulation. All three tested ckII 1 and ckII 2 knock-out lines showed no significant visible senescence phenotype, but chlorophyll loss was clearly delayed in the ckIIa 2 mutant plants. Furthermore, the analyses of the expression of the senescence-associated marker gene $S A G 12$ resulted in a strong decrease in all ckII $\alpha$ knock-out lines compared to the Col-0 wild type line indicating a delay in senescence on the molecular level. This is in line with findings of Mulekar and 
coworkers [33] who also detected no clear visible phenotypes in single mutants. However, the double and triple mutants are delayed in flowering and are affected in diverse developmental and stress responsive pathways rendering senescence phenotyping extremely difficult or even impossible; therefore, these lines were not included in our analyses. Due to gene redundancy, expression analyses of the direct target genes of GBF1 revealed that in the single ckIIa mutants not many differences are observed compared to wild type plants (Figure S6). No significant differences in the down-regulation of RBCS1 $a$ expression could be detected. Likewise, CAT2 downregulation is not altered in ckIIal deficient plants but impaired in ckII 2 mutants indicating that CKII might play a role in GBF1-mediated CAT2 downregulation, that the different $\alpha$ subunits appear to be involved to different extends and that CKII 2 deletion cannot be fully compensated (Figure S6). This is in agreement with the significant delay in chlorophyll loss and with a slower increase in hydrogen peroxide contents of the ckIIa2 deficient plants (Figure S7). However, these differences between the two direct target genes of GBF1 also indicate that GBF1 most likely operates not alone but in different higher order complexes on both promoters.

Taken together, our results indicate that phosphorylation is affecting DNA-binding of GBF1 and that CKII might be one of the kinases involved in the senescence-specific regulation of GBF1. We propose the following model how CKII can be involved in GBF1-mediated senescence regulation (Figure 5). We postulate that in young leaves, the CKII kinase activity is high since CKII is also involved in very basic cellular processes like cell cycle, DNA damage or translation, and is therefore, as already mentioned, not regulated by a simple on/off switch but more in a dynamic way. By a so far unknown trigger, either activity or protein stability or translation efficiency of CKII decrease at bolting time, leading to a non-phosphorylated state of GBF1 which in turn can bind with higher affinity to the promoter of CAT2 or RBCS1a leading to the repression of these genes. CAT2 repression will lead to an increase in hydrogen peroxide content which is used as a signal to induce senescence, whereas RBCSIa repression will lead to a decrease in carbon fixation which is also observed already early in senescence. It is so far unclear which trigger could be involved in the inactivation of CKII but there are several links between CKII and different hormones like salicylic acid, abscisic acid or auxin [49-51]. In a complete ckII $\alpha$ knock-out line it could be expected that GBF1 would not be phosphorylated at the CKII specific sites and CAT2 and $R B C S 1$ would be repressed all over development leading to high levels of hydrogen peroxide and low carbon fixation and thus very early senescence. However, this is not observed in the single mutants. If at all a delay of $S A G 12$ expression and for ckII 2 deficient plants also a delay in chlorophyll breakdown and hydrogen peroxide increase can be observed. Due to gene redundancy, no effects or only weak ones can be observed in the single mutants, but it can be speculated that the inactivation of specific $\alpha$ subunits might be more efficient than those of others so that in the ckIIa2 mutants inactivation of CKII 1 and 3 would be delayed leading also to a delay in senescence signaling. Moreover, high levels of hydrogen peroxide from early on can lead to compensatory effects like observed for cat 2 and cat 3 mutant plants. These plants neither exhibited increased levels of hydrogen peroxide nor showed accelerated senescence under normal long day conditions [52]. 


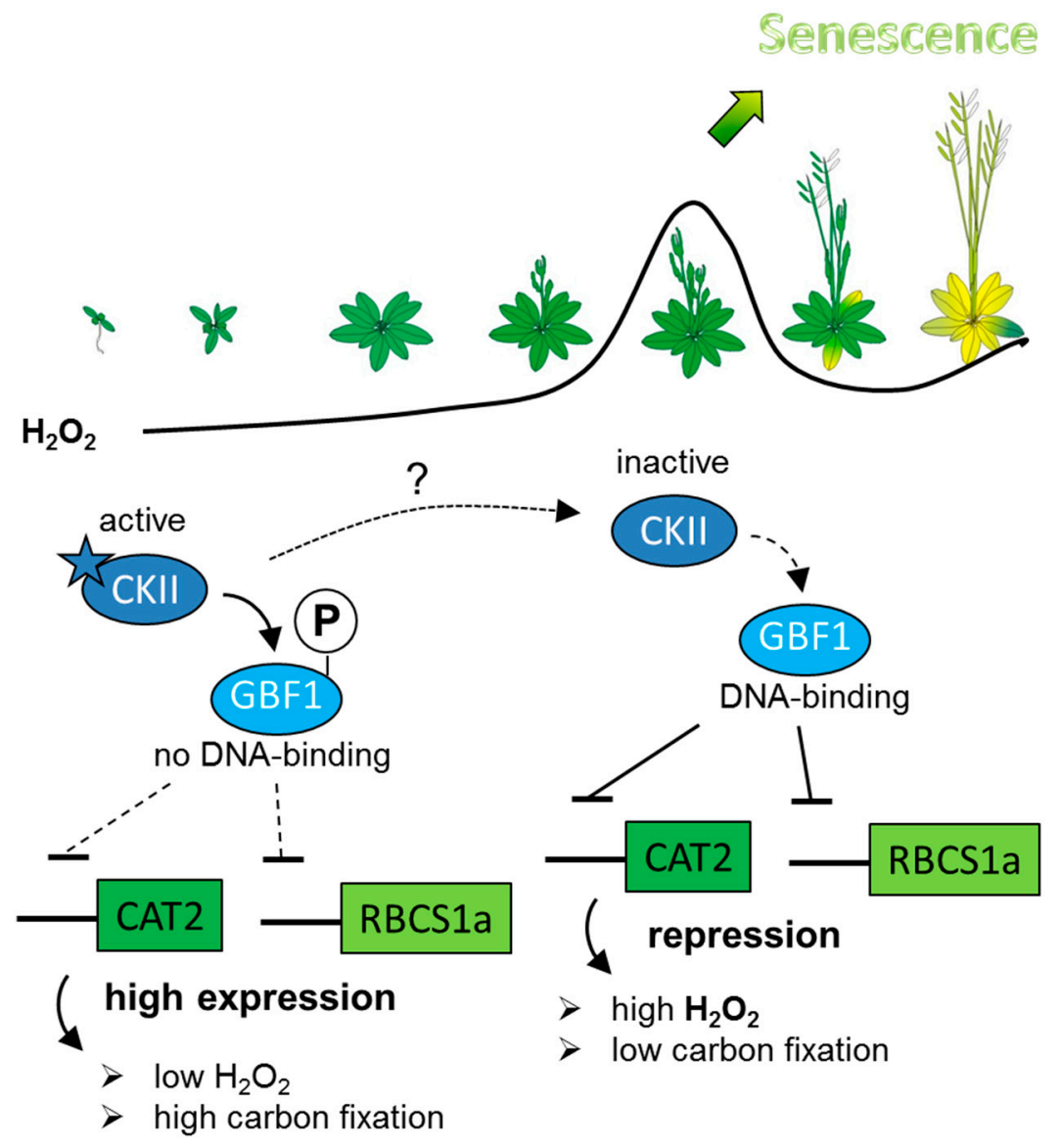

Figure 5. Model of senescence-specific activation of GBF1 by CKII phosphorylation postulating that CKII is active in young leaves and is inactivated during bolting time by a so far unknown trigger. Non-phosphorylated GBF1 can bind now to its target gene promoters $C A T 2$ and RBCS1a leading to the repression of these genes. Repression of CAT2 and RBSCS1a then leads to an increase in intracellular hydrogen peroxide contents and low carbon fixation rates. Hydrogen peroxide was shown to be a signal to induce senescence.

\section{Experimental Section}

\subsection{Plant Material}

Arabidopsis thaliana (L. Heynh), ecotype Columbia plants were grown in climatic chambers at $22{ }^{\circ} \mathrm{C}$ under long day conditions (16 h light/8 h dark) under moderate light intensity (60-80 $\mu \mathrm{mol} \mathrm{s}-1 \mathrm{~m}-2)$. During growth and development of the rosettes, the leaves were color-coded with different colored threads. This allows that individual leaves could be identified according to their age even in very late stages of development. Leaves of the same positions within different rosettes were pooled. Seeds of T-DNA insertion lines in CKIIal (SALK_021073 in exon 4; SALK_131097 in intron 1) and CKIIa2 (SALK_126662 in exon 10) were obtained from the Nottingham Arabidopsis Stock Centre (NASC) and were characterized by PCR to be homozygous for the T-DNA insertion using gene specific and T-DNA left border primers (LBb1). No expression of CKII 1 and CKII 2 was detected in the knock-out lines by RT-PCR, respectively. 


\subsection{Senescence Phenotyping}

For the evaluation of leaf senescence phenotypes leaves were sorted according to their age using a color code. In addition, leaves of at least five plants were categorized in four groups according to their leaf color: (1) "green"; (2) leaves starting to get yellow from the tip as "yellow-green"; (3) completely yellow leaves as "yellow" and (4) dry and/or brown leaves as "dry". Chlorophyll content was estimated using an atLEAF+ chlorophyll meter. Each leaf was measured in triplicate and values were averaged. Leaves No. 4 to 7 of each rosette were measured.

\subsection{Recombinant Proteins}

The full length GBF1 cDNA fragment or the mutated versions were cloned into the pDEST42 expression vector (Invitrogen/Thermo Fisher Scientific, Waltham, MA, USA) to create pDEST42-GBF1 encoding the $6 \times$ His-tagged protein. CKII 2 (AT3G50000) cDNA was also cloned into pDEST42 vector or pUC Spyne/Spyce for expression. After cultivation of $E$. coli cells carrying the expression plasmids in LB broth and $1 \mathrm{mM}$ IPTG treatment for induction of the recombinant protein syntheses, the cells were harvested, disrupted by sonication and centrifuged. The supernatants were used for experiments like Western blots, DPI-ELISAs and in vitro kinase assays. In vitro kinase assays were also conducted with purified proteins. Here, $500 \mathrm{~mL}$ of $E$. coli culture (BL21) expressing $6 \times$ His-tagged proteins was lysed in $2 \mathrm{~mL}$ LEW buffer (Macherey-Nagel, Düren, Germany), sonicated and centrifuged. The clear lysates were applied on Protino Ni-TED resin (Macherey-Nagel), washed with LEW buffer and eluted with elution buffer containing imidazol (Macherey-Nagel). The protein purification was verified by Western blot analyses followed by immune detection.

\subsection{Western Blot Analysis and Immune Detection of $6 \times$ His-Tagged GBF1}

$10 \%$ SDS polyacrylamide gels were used for protein separation and were subsequently stained with Coomassie brilliant blue $R-250$ solution $(B I O-R A D)$ or transferred to nitrocellulose membranes using a semi-dry blotting procedure. After blocking for $1 \mathrm{~h}$ at room temperature in TBS-T $(150 \mathrm{mM} \mathrm{NaCl}$, $50 \mathrm{mM}$ Tris, $\mathrm{pH} 7.6,0.5 \%$ Tween-20) containing 5\% (w/v) non-fat dry milk powder, the filters were gently shaken for $1 \mathrm{~h}$ in Anti-His antibodies solution (Qiagen, Hilden, Germany). Filters were washed three times in TBST for $10 \mathrm{~min}$ and then incubated with the secondary antibodies coupled with a horse radish peroxidase (Cell Signalling Technology/New England Biolabs GmbH, Leiden, The Netherlands). Again, the filters were washed 3 times in TBS-T for $10 \mathrm{~min}$. Subsequently, antibody conjugates were detected by chemo luminescence. Quantification of the detected proteins was performed using the ImageQuantTL software (GE Healthcare Life Sciences, Cleveland, OH, USA).

\subsection{DPI-ELISA}

$100 \mathrm{~mL}$ culture of $E$. coli cells (BL21) expressing $6 \times$ His-tagged GBF1 or mutated versions of GBF 1 were centrifuged and lysed in $1 \mathrm{~mL}$ of lyses buffer ( $50 \mathrm{mM}$ Tris, $\mathrm{pH} 7.5,50 \mathrm{mM} \mathrm{NaCl}, 5 \mathrm{mM} \mathrm{MgCl} 2,1$ $\mathrm{mM}$ PMSF, $0.02 \% \mathrm{NP} 40$ ). Using this bacterial protein extract the DNA-binding assay was performed as described by Kirchler and coworkers [25]. Streptavidin coated ELISA wells (Nunc Immobilizer) were loaded with 2 pmol of the biotinylated CAT2 promoter fragment containing the wild type (5' 
ctcatcacacgtggaatcc $\left.3^{\prime}\right)$ or mutated G-box (5' ctcatcaaaaaaagaatcc $\left.3^{\prime}\right)$ or a $R B C S I$ a promoter fragment also containing a G-Box (5' aattatcttccacgtggcattattcc $3^{\prime}$ ) or a mutated version ( $5^{\prime}$ aattatcttcceagtggcattattcc $3^{\prime}$ ). These ELISA plates were blocked with 1\% blocking agent (Roche, Mannheim, Germany). $2 \mu \mathrm{L}$ of crude protein extracts $(5 \mu \mathrm{g} / \mu \mathrm{L})$ was diluted 1:50, 1:100, 1:200 in binding puffer and $60 \mu \mathrm{L}$ were added to each well and incubated for $1 \mathrm{~h}$. Subsequently, the raw lysate was removed and the wells were washed with blocking buffer (Qiagen) containing 0.1\% Tween 20. After removing the washing buffer each well was incubated for $1 \mathrm{~h}$ with Anti-His antibody conjugates (Penta-His HRP, Qiagen) at room temperature. The OPD substrate (1,2-phenylenediamine hydrochlorid, Dako, Glostrup, Denmark) was added to the wells after intensive washing with blocking buffer (Qiagen), The detection reaction of the DNA-bound proteins was performed following the manufacturer's protocol (Dako). Subsequently, the plates were read out at an absorbance of $492 \mathrm{~nm}$ using an ELISA plate reader (Tecan, Männedorf, Switzerland with filter setting at $650 \mathrm{~nm}$ for reference). Mammalian CKII and recombinant plant CKII were added to the 1:50 diluted protein extracts prior to DNA-binding and incubated for $30 \mathrm{~min}$ at $30{ }^{\circ} \mathrm{C}$.

\subsection{Site-Directed Mutagenesis}

Site directed mutagenesis was performed following the protocol of the QuickChange II Site-Directed Mutagenesis Kit (Stratagene/Agilent Technologies, Santa Clara, CA, USA). As DNA template, the wild type version of $G B F 1$ was used. The primers were designed according to the manufacture's advice (Table S1). Successful mutagenesis was verified by sequencing.

\subsection{In Vitro Kinase Assay}

Phosphorylation activities of mammalian Casein Kinase II (CKII, NEB) was assayed at $30{ }^{\circ} \mathrm{C}$ for $30 \mathrm{~min}$ in a final volume of $20 \mu \mathrm{L}$ reaction buffer $(10 \times$ Kinase Buffer, NEB) containing $100 \mu \mathrm{M}$ ATP and $50 \mu \mathrm{Ci}\left[\gamma_{-}{ }^{32} \mathrm{P}\right] \mathrm{ATP}\left(5000 \mathrm{Ci} \mathrm{mmol}^{-1}\right)$ and one of the substrates with the protein amount of approx. 15 or $45 \mu \mathrm{g}$. The kinase assay with purified GBF1 proteins was conducted using a final protein amount of 0.5 $\mu \mathrm{g}$. Casein (25-35 kDa, Sigma-Aldrich, St. Louis, MO, USA) was used as a control protein. The reaction was terminated by the addition of $4 \times$ SDS sample loading buffer. After electrophoresis on $10 \%(w / v)$ SDS-PAGE, the phosphorylated substrates were visualized by autoradiography.

\subsection{Semi-quantitative RT PCR}

RNA was isolated from pooled leaf material using leaf No. 6 and 7 with the PURESCRIPT RNA Isolation Kit (Gentra, Biozym). cDNA was polymerized using the iScript ${ }^{\mathrm{TM}} \mathrm{cDNA}^{\mathrm{S}}$ Synthesis Kit (Bio-Rad, Munich, Germany) according to the manufacturer's instructions. PCR reactions were performed on cDNA using different primer pairs (see Table 1) and a low cycle number (25 cycles) in which the PCR did not yet reach the saturation phase. Subsequently, RT-PCR products were separated on $1 \%$ agarose gels. The band intensity was quantified using the NIH Image program Scion Image (Scion Corporation). Expression was normalized to $A C T I N 2$ as reference gene for senescence since the variation of $A C T I N 2$ expression over different leaf and plant stages in Arabidopsis was very low in contrast to other housekeeping genes [53]. 
Table 1. Primers used for qRT-PCR.

\begin{tabular}{ccc}
\hline & Forward Primer 5'-3' & Reverse Primer $\mathbf{5}^{\prime} \mathbf{-} \mathbf{3}^{\prime}$ \\
\hline GBF1 & ggtcgaaagatggtgaagga & atccgattccaatcacgaag \\
CKIIa1 & ttgatccacaactggaagca & cattaccatcatcatcatcatcag \\
CKIIa2 & gcatttggtctcacctgagg & gaaaccggagggagtaataagaa \\
SAG12 & cccggttaatgatgagcaagc & gctttcatggcaagaccaca \\
CAT2 & caggttcgtcatgctgagaag & ttagatgcttggtctcacgtt \\
RBCS1a & attgcctacaagccaccaag & atttgtagccgcattgtcct \\
WRKY53 & gatcacaagaacaccaccattagcc & aaagttgtgtcaatctcgaccgttg \\
\hline
\end{tabular}

\subsection{Intracellular Hydrogen Peroxide Measurements}

Hydrogen peroxide content was determined as described in Smykowski et al. [19]. Whole Arabidopsis leaves were detached and incubated for $45 \mathrm{~min} 10 \mu \mathrm{M}$ 2'7'dichlorohydrofluorescein diacetate (DCFH-DA, Invitrogen/Thermo Fisher Scientific) at room temperature. Leaves were removed and rinsed in water, dried with filter paper and then frozen in liquid nitrogen. This material was either immediately ground or stored for a maximum of two weeks. Leaf tissue was homogenized in $1 \mathrm{~mL}$ of $40 \mathrm{mM}$ Tris-HCI buffer $\mathrm{pH} 7.0$, centrifuged at 20,600 $\mathrm{g}$ for $15 \mathrm{~min}$ and the supernatant was recovered. Fluorescence was determined using a spectrofluorometer (Berthold) using an excitation wavelength of $488 \mathrm{~nm}$ and an emission wavelength of $525 \mathrm{~nm}$.

\section{Conclusions}

One of the major regulatory mechanisms governing plant senescence is transcriptional reprogramming governed by a high number of different transcription factors. Many of the senescence-regulating transcription factors are themselves induced or repressed on the transcriptional level; however, at some point, several transcription factors have to be regulated on the posttranscriptional level and GBF1 belongs to these factors. One possible regulatory mechanism is the activation or inactivation by phosphorylation by different kinases and CKII appears to be one of the senescence-regulating kinases modulating the DNA-binding activity of GBF1. But how the activity of CKII is controlled during development and onset of senescence and which other kinases are involved in the modulation of GBF1 activity still has to be elucidated.

\section{Acknowledgments}

We thank Gesine Seibold and Gabriele Eggers-Schumacher for their excellent technical assistance. We thank Kenneth Berentzen for critically reading the manuscript. We thank NASC for providing seeds of T-DNA insertion lines in CKIIal (SALK_021073; SALK_131097) and CKIIa2 (SALK_126662). This research was supported by the European Union (FP7, ITN MERIT) and the Deutsche Forschungsgemeinschaft (ZE 313/8-2, ZE 313/9-1, SFB 1101/B06).

\section{Author Contributions}

Ulrike Zentgraf conceived and coordinated the experiments; Anja Smykowski did the kinase assays with CKII, the phenotyping, the hydrogen peroxide measurements and the RT-PCRs. Stefan M. Fischer 
and Anja Smykowski did the site-directed mutagenesis and the DPI-ELISAs with the mutated GBF1 versions. Ulrike Zentgraf and Anja Smykowski wrote the manuscript. All authors contributed to finalizing the manuscript.

\section{Conflicts of Interest}

The authors declare no conflicts of interest.

\section{References}

1. Balazadeh, S.; Riano-Pachon, D.M.; Mueller-Roeber, B. Transcription factors regulating leaf senescence in Arabidopsis thaliana. Plant Biol. 2008, 10, S63-S75.

2. Zentgraf, U.; Jobst, J.; Kolb, D.; Rentsch, D. Senescence-related gene expression profiles of rosette leaves of Arabidopsis thaliana: Leaf age versus plant age. Plant Biol. 2004, 6, 178-183.

3. Breeze, E.; Harrison, E.; McHattie, S.; Hughes, L.; Hickman, R.; Hill, C.; Kiddle, S.; Kim, Y.S.; Penfold, C.A.; Jenkins, D.; et al. High-resolution temporal profiling of transcripts during Arabidopsis leaf senescence reveals a distinct chronology of processes and regulation. Plant Cell 2011, 23, 873-894.

4. Buchanan-Wollaston, V.; Page, T.; Harrison, E.; Breeze, E.; Lim, P.O.; Nam, H.G.; Lin, J.F.; Wu, S.H.; Swidzinski, J.; Ishizaki, K.; et al. Comparative transcriptome analysis reveals significant differences in gene expression and signalling pathways between developmental and dark/starvation-induced senescence in Arabidopsis. Plant J. 2005, 42, 567-585.

5. Guo, Y.; Cai, Z.; Gan, S. Transcriptome of Arabidopsis leaf senescence. Plant Cell Environ. 2004, 27, 521-549.

6. Balazadeh, S.; Siddiqui, H.; Allu, A.D.; Matallana-Ramirez, L.P.; Caldana, C.; Mehrnia, M.; Zanor, M.I.; Köhler, B.; Mueller-Roeber, B. A gene regulatory network controlled by the NAC transcription factor ANAC092/AtNAC2/ORE1 during salt-promoted senescence. Plant J. 2010, 62, 250-264.

7. Balazadeh, S.; Kwasniewski, M.; Caldana, C.; Mehrnia, M.; Zanor, M.I.; Mueller-Roeber, B.; Xue, G.P. ORS1, an $\mathrm{H}_{2} \mathrm{O}_{2}$-responsive NAC transcription factor, controls senescence in Arabidopsis thaliana. Mol. Plant 2011, 4, 346-360.

8. Zentgraf, U.; Laun, T.; Miao, Y. The complex regulation of WRKY53 during leaf senescence of Arabidopsis thaliana. Eur. J. Cell Biol. 2010, 89, 133-137.

9. Xie, Y.; Huhn, K.; Brandt, R.; Potschin, M.; Bieker, S.; Straub, D.; Doll, J.; Drechsler, T.; Zentgraf, U.; Wenkel, S. REVOLUTA and WRKY53 connect early and late leaf development in Arabidopsis. Development 2014, 141, 4772-4783.

10. Miao, Y.; Laun, T.; Zimmermann, P.; Zentgraf, U. Targets of the WRKY53 transcription factor and its role during leaf senescence in Arabidopsis. Plant Mol.Biol.2004, 55, 853-867.

11. Wu, A.; Allu, A.D.; Garapati, P.; Siddiqui, H.; Dortay, H.; Asensi-Fabado, M.A.; Munné-Bosch, S.; Zanor, M.I.; Antonio, C.; Tohge, T.; et al. JUNGBRUNNEN1, a reactive oxygen species-responsive NAC transcription factor, regulates longevity in Arabidopsis. Plant Cell 2012, 24, 482-506.

12. Uauy, C.; Distelfeld, A.; Fahima, T.; Blechl, A.; Dubcovsky, J. A NAC gene regulating senescence improves grain protein, zinc, and iron content in wheat. Science 2006, 314, 1298-1301. 
13. Ulker, B.; Shahid Mukhtar, M.; Somssich, I.E. The WRKY70 transcription factor of Arabidopsis influences both the plant senescence and defense signaling pathways. Planta 2007, 226, 125-137.

14. Besseau, S.; Li, J.; Palva, E.T. WRKY54 and WRKY70 co-operate as negative regulators of leaf senescence in Arabidopsis thaliana. J. Exp. Bot. 2012, 63, 2667-2679.

15. Zimmermann, P.; Heinlein, C.; Orendi, G.; Zentgraf, U. Senescence-specific regulation of catalases in Arabidopsis thaliana (L.) Heynh. Plant Cell Environ. 2006, 29, 1049-1060.

16. Bieker, S.; Riester, L.; Stahl, M.; Franzaring, J.; Zentgraf, U. Senescence-specific alteration of hydrogen peroxide levels in Arabidopsis thaliana and oilseed rape spring variety Brassica napus L. cv. Mozart. J. Integr. Plant Biol. 2012, 54, 540-554.

17. Agüera, E.; Cabello, P.; de la Haba, P. Induction of leaf senescence by low nitrogen nutrition in sunflower (Helianthus annuus) plants. Physiol. Plant. 2010, 138, 256-267.

18. Ye, Z.; Rodriguez, R.; Tran, A.; Hoang, H.; de los Santos, D.; Brown, S.; Vellanoweth, R.L. The developmental transition to flowering represses ascorbate peroxidase activity and induces enzymatic lipid peroxidation in leaf tissue in Arabidopsis thaliana. Plant Sci. 2000, 158, 115-127.

19. Smykowski, A.; Zimmermann, P.; Zentgraf, U. G-Box binding Factor1 reduces CATALASE2 expression and regulates the onset of leaf senescence in Arabidopsis. Plant Physiol. 2010, 153, $1321-1331$.

20. Jakoby, M.; Weisshaar, B.; Dröge-Laser, W.; Vicente-Carbajosa, J.; Tiedemann, J.; Kroj, T.; Parcy, F. bZIP transcription factors in Arabidopsis. Trends Plant Sci. 2002, 7, 106-111.

21. Foster, R.; Izawa, T.; Chua, N.H. Plant bZIP proteins gather at ACGT elements. FASEB J. 1994, 8, 192-200.

22. Izawa, T.; Foster, R.; Chua, N.H. Plant bZIP protein DNA binding specificity. J. Mol. Biol. 1993, 230, 1131-1144.

23. Menkens, A.E.; Schindler, U.; Cashmore, A.R. The G-box: A ubiquitous regulatory DNA element in plants bound by the GBF family of bZIP proteins. Trends Biochem. Sci. 1995, 20, 506-510.

24. Hardtke, C.S.; Gohda, K.; Osterlund, M.T.; Oyama, T.; Okada, K.; Deng, X.W. HY5 stability and activity in Arabidopsis is regulated by phosphorylation in its COP1 binding domain. EMBOJ. 2000, 19, 4997-5006.

25. Kirchler, T.; Briesemeister, S.; Singer, M.; Schutze, K.; Keinath, M.; Kohlbacher, O.; Teige, M.; Vicente-Carbajosa, J.; Harter, K.; Chaban, C. The role of phosphorylatable serine residues in the DNA-binding domain of Arabidopsis bZIP transcription factors. Eur. J. Cell Biol. 2010, 89, 175-183.

26. Siberil, Y.; Doireau, P.; Gantet, P. Plant bZIP G-box binding factors. Modular structure and activation mechanisms. Eur. J. Biochem. 2001, 268, 5655-5666.

27. Terzaghi, W.B.; Bertekap, R.L., Jr.; Cashmore, A.R. Intracellular localization of GBF proteins and blue light-induced import of GBF2 fusion proteins into the nucleus of cultured Arabidopsis and soybean cells. Plant J. 1997, 11, 967-982.

28. Klimczak, L.J.; Schindler, U.; Cashmore, A.R. DNA binding activity of the Arabidopsis G-box binding factor GBF1 is stimulated by phosphorylation by casein kinase II from broccoli. Plant Cell 1992, 4, 87-98. 
29. Droge-Laser, W.; Kaiser, A.; Lindsay, W.P.; Halkier, B.A.; Loake, G.J.; Doerner, P.; Dixon, R.A.; Lamb, C. Rapid stimulation of a soybean protein-serine kinase that phosphorylates a novel bZIP DNA-binding protein, $\mathrm{G} / \mathrm{HBF}-1$, during the induction of early transcription-dependent defenses. EMBO J. 1997, 16, 726-738.

30. Ciceri, P.; Gianazza, E.; Lazzari, B.; Lippoli, G.; Genga, A.; Hoscheck, G.; Schmidt, R.J.; Viotti, A. Phosphorylation of Opaque2 changes diurnally and impacts its DNA binding activity. Plant Cell 1997, 9, 97-108.

31. Meshi, T.; Moda, I.; Minami, M.; Okanami, M.; Iwabuchi, M. Conserved Ser residues in the basic region of the bZIP-type transcription factor HBP-1a(17): Importance in DNA binding and possible targets for phosphorylation. Plant Mol. Biol. 1998, 36, 125-136.

32. Shen, W.H.; Gigot, C. Protein complexes binding to cis elements of the plant histone gene promoters: Multiplicity, phosphorylation and cell cycle alteration. Plant Mol. Biol. 1997, 33, 367-379.

33. Mulekar, J.J.; Bu, Q.; Chen, F.; Huq, E. Casein kinase II $\alpha$ subunits affect multiple developmental and stress-responsive pathways in Arabidopsis. Plant J. 2012, 69, 343-354.

34. Moreno-Romero, J.; Espunya, C.; Platara, M.; Arino, J.; Martinez, M.C. A role for protein kinase CK2 in plant development: Evidence obtained using a dominant-negative mutant. Plant J. 2008, $55,118-130$.

35. Mulekar, J.J.; Huq, E. Expanding roles of protein kinase CK2 in regulating plant growth and development. J. Exp. Bot. 2014, 65, 2883-2893.

36. Salinas, P.; Fuentes, D.; Vidal, E.; Jordana, X.; Echeverria, M.; Holuigue, L. An extensive survey of CK2 alpha and beta subunits in Arabidopsis: Multiple isoforms exhibit differential subcellular localization. Plant Cell Physiol. 2006, 47, 1295-1308.

37. Schweer, J.; Türkeri, H.; Link, B.; Link, G. AtSIG6, a plastid sigma factor from Arabidopsis, reveals functional impact of cpCK2 phosphorylation. Plant J. 2010, 62, 192-202.

38. Kang, H.-G.; Klessig, D.F. Salicylic acid-inducible Arabidopsis CK2-like activity phosphorylates TGA2. Plant Mol. Biol. 2005, 57, 541-557.

39. Nieva, C.; Busk, P.K.; Domínguez-Puigjaner, E.; Lumbreras, V.; Testillano, P.S.; Risueño, M.-C.; Pagès, M. Isolation and functional characterisation of two new bZIP maize regulators of the ABA responsive gene rab28. Plant Mol. Biol. 2005, 58, 899-914.

40. Collinge, M.A.; Walker, J.C. Isolation of an Arabidopsis thaliana casein kinase II beta subunit by complementation in Saccharomyces cerevisiae. Plant Mol. Biol. 1994, 25, 649-658.

41. Dobrowolska, G.; Meggio, F.; Szczegielniak, J.; Muszynska, G.; Pinna, L.A. Purification and characterization of maize seedling casein kinase IIB, a monomeric enzyme immunologically related to the alpha subunit of animal casein kinase-2. Eur. J. Biochem. 1992, 204, 299-303.

42. Schindler, U.; Menkens, A.E.; Beckmann, H.; Ecker, J.R.; Cashmore, A.R. Heterodimerization between light-regulated and ubiquitously expressed Arabidopsis GBF bZIP proteins. EMBO J. 1992, 11, 1261-1273.

43. Meggio, F.; Pinna, L.A. One-thousand-and-one substrates of protein kinase CK2? FASEB J. 2003, 17, 349-368.

44. Klimczak, L.J.; Collinge, M.A.; Farini, D.; Giuliano, G.; Walker, J.C.; Cashmore, A.R. Reconstitution of Arabidopsis casein kinase II from recombinant subunits and phosphorylation of transcription factor GBF1. Plant Cell 1995, 7, 105-115. 
45. Berberich, S.J.; Cole, M.D. Casein kinase II inhibits the DNA-binding activity of Max homodimers but not Myc/Max heterodimers. Genes Dev. 1992, 6, 166-176.

46. Ellenberger, T.E.; Brandl, C.J.; Struhl, K.; Harrison, S.C. The GCN4 basic region leucine zipper binds DNA as a dimer of uninterrupted alpha helices: Crystal structure of the protein-DNA complex. Cell 1992, 71, 1223-1237.

47. Kohler, J.J.; Metallo, S.J.; Schneider, T.L.; Schepartz, A. DNA specificity enhanced by sequential binding of protein monomers. Proc. Natl. Acad. Sci. USA 1999, 96, 11735-11739.

48. Metallo, S.J.; Schepartz, A. Certain bZIP peptides bind DNA sequentially as monomers and dimerize on the DNA. Nat. Struct. Biol. 1997, 4, 115-117.

49. Moreno-Romero, J.; Armengot, L.; Marquès-Bueno, M.M.; Cadavid-Ordóñez, M.; Martínez, M.C. About the role of CK2 in plant signal transduction. Mol. Cell. Biochem. 2011, 356, 233-240.

50. Armengot, L.; Marquès-Bueno, M.M.; Soria-Garcia, A.; Müller, M.; Munné-Bosch, S.; Martínez, M.C. Functional interplay between protein kinase CK2 and salicylic acid sustains PIN transcriptional expression and root development. Plant J. 2014, 78, 411-423.

51. Wang, Y.; Chang, H.; Hu, S.; Lu, X.; Yuan, C.; Zhang, C.; Wang, P.; Xiao, W.; Xiao, L.; Xue, G.P.; et al. Plastid casein kinase 2 knockout reduces abscisic acid (ABA) sensitivity, thermotolerance, and expression of ABA- and heat-stress-responsive nuclear genes. J. Exp. Bot. 2014, 65, 4159-4175.

52. Zentgraf, U.; Zimmermann, P.; Smykowski, A. Role of intracellular hydrogen peroxide as signaling molecule for plant senescence. In Senescence; Nagata, T., Ed.; In-tech Open Access Publishing: Rijeka, Croatia, 2012; doi:10.5772/34576.

53. Panchuk, I.I.; Zentgraf, U.; Volkov, R.A. Expression of the APX gene family during leaf senescence of Arabidopsis thaliana. Planta 2005, 222, 926-932.

(C) 2015 by the authors; licensee MDPI, Basel, Switzerland. This article is an open access article distributed under the terms and conditions of the Creative Commons Attribution license (http://creativecommons.org/licenses/by/4.0/). 